Pacific Journal of Mathematics

MEROMORPHIC ANNULAR FUNCTIONS 


\title{
MEROMORPHIC ANNULAR FUNCTIONS
}

\author{
JOSEPH WARREN
}

The purpose of this paper is to present a definition of meromorphic annular functions which includes the definition of holomorphic annular functions. Several equivalent conditions for meromorphic annular functions are given.

2. Preliminary definitions and remarks. Let $D$ be the disk $|z|<1$ and $C$ the circle $|z|=1$. We shall, henceforth, assume that the function $f(z)$ is meromorphic in $D$.

A boundary path in $D$ is the image of the unit interval $0 \leqq t<1$ under a continuous function $z=z(t)$ from $0 \leqq t<1$ into $D$ such that $\lim _{t \rightarrow 1}|z(t)|=1$. A spiral in $D$ is a boundary path with the additional condition that $\lim _{t \rightarrow 1} \arg z(t)=+\infty$ or $-\infty$ for any branch of the argument of $z(t)$.

The set $L(\lambda)=\{z|f(z)|=\lambda, 0<\lambda<\infty\}$ is called a level set for the function $f$ and a component of $L(\lambda)$ is called a level curve. It is known [6, Prop. 1] that if $C(\lambda)$ is a level curve which does not contain any zeros of $f^{\prime}(z)$, then $C(\lambda)$ is either a Jordan curve contained in $D$ or the union of two disjoint boundary paths. If $\lambda=0$ or $\lambda=\infty$, then $L(\lambda)$ corresponds to the set of zeros or poles, respectively, of $f(z)$.

The function $f(z)$ has the asymptotic value a (allowing $a=\infty$ ) if there exists a boundary path $z=z(t), 0 \leqq t<1$, such that $\lim _{t \rightarrow 1}$ $f(z(t))=a$.

The following definition will be taken for the definition of meromorphic annular functions.

DEFINITION 1. Let $f(z)$ be a nonconstant meromorphic function in $D$ and let $\left\{J_{n}\right\}$ be a sequence of Jordan curves with $J_{1}$ contained in the interior of $J_{n}$ for $n=2,3,4, \cdots$ such that either

$$
\lim _{n \rightarrow \infty z \in J_{n}} \max _{n}|f(z)-a|=0,
$$

for a finite value $a$, or, if $a=\infty$,

$$
\lim _{n \rightarrow \infty} \min _{n}|f(z)|=\infty .
$$

If, in addition, $f$ has an asymptotic value, then $f$ will be called an annular function with respect to $a$.

The class of annular functions with respect to $a$ will be denoted by $\mathscr{A}(a)$. 
REMARK 1. It is proved in Theorem 1 of [7] that if $f \in \mathscr{A}(a)$, then given any $r, 0<r<1$, there exists an integer $N$ such that if $n \geqq N$, then the disk $|z| \leqq r$ is contained in the interior of $J_{n}$. In such a case the sequence $\left\{J_{n}\right\}$ is said to converge uniformly to the boundary $C$.

REMARK 2. A subsequence $\left\{J_{n_{k}}\right\}$ can be selected such that if $k \neq j$ then $J_{n_{k}} \cap J_{n_{j}}=\phi$.

From these two remarks it may be assumed that the members of the sequence $\left\{J_{n}\right\}$ of Definition 1 are pairwise disjoint and that the sequence tends uniformly to $C$.

REMARK 3. It is evident that if $f \in \mathscr{A}(a)$ then the asymptotic value assumed to exist in Definition 1 is $a$.

REMARK 4. If $a \neq b$, then $\mathscr{A}(a) \cap \mathscr{A}(b)=\phi$. The function $f$ is an $\mathscr{A}(a)$ if and only if $1 / f \in \mathscr{A}(1 / a)$.

REMARK 5. If $f$ is holomorphic and annular in the old sense $[1,340]$ then there exists a sequence of Jordan curves $\left\{J_{n}\right\}$ which tend to the circle $C$ and on which $f$ tends uniformly to $\infty$. Since every holomorphic function has an asymptotic value, which in this case must be $\infty$, it is seen that $f \in \mathscr{A}(\infty)$. Thus there exists a function in $\mathscr{A}(0)$; the reciprocal of any function annular in the old sense.

The following definitions are needed.

Definition 2. If the nonconstant meromorphic function $f$ in $D$ has the asymptotic value $a$ on a spiral asymptotic path, then $f$ is a spiral function with respect to $a$.

The class of spiral function with respect to $a$ will be denoted by $\mathscr{S}(a)$.

DEFINITION 3. If the nonconstant and meromorphic function $f$ in $D$ is bounded away from $a$ on a spiral boundary path, then $f$ is said to be in the Valiron class with respect to $a$, provided $f$ has the asymptotic value $a$.

The class of such functions will be denoted by $\mathscr{V}(a)$.

REMARK 6. $\mathscr{\mathscr { S }}(\alpha) \subset \mathscr{S}(\alpha)$.

Definition 4. The function $f(z)$ is in the class $\mathscr{L}^{\prime}(a)$ if $f$ is nonconstant and meromorphic in $D$ and has the asymptotic value $a$ as well as the following property: In the case of a finite value $a$, 
every level curve of $f(z)-a$ which is disjoint from the zeros of $f^{\prime}(z)$ is a compact set in $D$, or, in the case of $a=\infty$, every level curve of $f(z)$ which is disjoint from the zeros of $f^{\prime}(z)$ is a compact set in $D$.

Definition 5. Let $\mathscr{L}(a)$ be the class of functions $f$ such that $f$ is in $\mathscr{L}^{\prime}(\alpha)$ and such that every level curve of $f(z)-a$ (or $f(z)$ if $a=\infty$ ) is a compact set in $D$.

It will be shown that $\mathscr{L}(a)=\mathscr{L}^{\prime}(a)$.

Definition 6. A tract $\{D(\epsilon), a\}$ for the meromorphic function $f$ in $D$ associated with the value $a$ is a set of non-void domains $D(\varepsilon)$ each of which is a component of $\{z|| f(z)-a \mid<\varepsilon\}$, or $\{z|| f(z) \mid>1 / \varepsilon\}$ if $a=\infty$, such that $D(\varepsilon) \subset D\left(\varepsilon^{\prime}\right)$ if $\varepsilon<\varepsilon^{\prime}$ and $\bigcap_{\varepsilon<0} D(\varepsilon)=\phi$.

3. Equivalences for $\mathscr{A}(a)$. The following theorem gives the main equivalences for the class $\mathscr{A}(\alpha)$ and corresponds to Theorems 1 and 3 of [6].

THEOREM 1. $\mathscr{A}(a)=\mathscr{S}(a)-\mathscr{V}(\alpha)=\mathscr{L}^{\prime}(\alpha)=\mathscr{L}(a)$.

Proof. To prove the theorem in the most economical way we prove the chain of containments $\mathscr{A}(a) \subset \mathscr{S}(a)-\mathscr{Y}(a) \subset \mathscr{L}^{\prime}(a) \subset$ $\mathscr{A}(a) \subset \mathscr{S}(a) \subset \mathscr{L}^{\prime}(a)$.

First, let $f \in \mathscr{A}(a)$, let $T$ be the asymptotic path on which $f$ tends to a (see Remark 3), and let $\left\{J_{n}\right\}$ be the sequence of Jordan curves of Definition 1 on which $f$ tends uniformly to $a$. Using the same construction as in Theorem 2 of [6] a spiral may be constructed on which $f$ has the asymptotic value $a$. Thus $f \in \mathscr{S}(a)$. Evidently every boundary path intersects members of $\left\{J_{n}\right\}$ for all sufficiently large $n$ so that $f$ cannot be bounded away from $a$ on any spiral. Since $f$ has the asymptotic value $a, f$ is not in $\mathscr{V}(a)$ and is in $\mathscr{S}(a)$ $-\mathscr{Y}(a)$.

Now let $f \in \mathscr{S}(a)$ and let $C(\lambda)$ be a level curve of $f(z)-a$ which contains no zeros of $f^{\prime}(z)$. If $C(\lambda)$ is not a Jordan curve in $D$, then it consists of two boundary paths (spirals) on which $f$ is bounded away from $\alpha$, and we may conclude that $f \in \mathscr{Y}(\alpha)$. Therefore, if $f \in \mathscr{S}(\alpha)-\mathscr{Y}(a)$, then each level curve $C(\lambda)$ of $f(z)-a$ containing no zeros of $f^{\prime}(z)$ must be a Jordan curve in $D$, and hence $f \in \mathscr{L}^{\prime}(a)$ and we obtain $\mathscr{S}(\alpha)-\mathscr{Y}(\alpha) \subset \mathscr{L}^{\prime}(a)$.

Let $f \in \mathscr{L}^{\prime}(a)$. Because $f$ has the asymptotic value $a$, there is a tract $\{D(\varepsilon), a\}$ associated with $\alpha$. Choose a sequence $\left\{\varepsilon_{n}\right\}$ of positive numbers such that $\varepsilon_{n} \downarrow 0$ as $n \rightarrow \infty$ and the level set $\{z|| f(z)-a \mid=$ $\left.\varepsilon_{n}\right\}$, or $\left\{z|| f(z) \mid=1 / \varepsilon_{n}\right\}$ if $a=\infty$, does not contain any zeros of $f^{\prime}(z)$ 
for $n=1,2, \cdots$.

For each $n, D\left(\varepsilon_{n}\right)$ contains an asymptotic path with asymptotic value $a$ so that it cannot be contained within a Jordan curve in $D$. Thus the set $D\left(\varepsilon_{n}\right)$ is $|z|<1$ with a countable or finite number of Jordan domains removed. Otherwise the boundary of $D\left(\varepsilon_{n}\right)$ would contain a level curve which is not a compact set in $D$, contrary to the hypothesis that $f \in \mathscr{L}^{\prime}(a)$.

If $D\left(\varepsilon_{n}\right)=D$ for every $n$ then $|f(z)-a|<\varepsilon_{n}$ (or $|f(z)|>1 / \varepsilon_{n}$ ) in $D$ for every $n$ and $f(z)$ is identically constant contrary to the definition of $\mathscr{L}^{\prime}(a)$. Thus there exists an integer $n_{1}$ and a point $z_{1} \in D$ which is not in $D\left(\varepsilon_{n_{1}}\right)$. Let $J_{1}$ be the Jordan curve in $D$ which contains $z_{1}$ in its interior and which is a component of the boundary of $D\left(\varepsilon_{n_{1}}\right)$. Let $J_{2}$ be the Jordan curve which contains $z_{1}$ in its interior and is a boundary component of $D\left(\varepsilon_{n_{1}+1}\right)$. Because of the definition of tract, $D\left(\varepsilon_{n_{1}+1}\right) \subset D\left(\varepsilon_{n_{1}}\right)$ which implies that $J_{2}$ contains $J_{1}$ in its interior. Continuing in the same manner we obtain a sequence of Jordan curves $\left\{J_{n}\right\}$ such that $J_{n}$ contains $J_{1}$ in its interior for $n=2,3,4, \cdots$ and such that $|f(z)-a|=\varepsilon_{n}$ (or $|f(z)|=1 / \varepsilon_{n}$ ) for all $z \in J_{n}, n=1,2, \cdots$. Since $f$ is not a constant, $J_{n}$ tends uniformly to $C$, or $\min _{z \in J_{n}}|z| \rightarrow 1$ as $n \rightarrow \infty$, because of [7, Theorem 1]. Thus $f$ has an asymptotic value and has the sequence $\left\{J_{n}\right\}$ with all the properties of Definition 1 so that $f$ is in $\mathscr{A}(a)$.

If $f \in \mathscr{A}(a)$, then it is easy to see that any level curve of $f(z)-a$ is contained inside one of the Jordan curves $J_{n}$ of Definition 1 and is thus compact in $D$. Thus $f \in \mathscr{L}(a)$.

Finally, $\mathscr{L}(a) \subset \mathscr{L}^{\prime}(a)$ by definition, and the proof of Theorem 1 is complete. There is one other characterization of the set $\mathscr{A}(a)$ which was suggested to me by J. Choike.

CoRollary. The function $f$ is in $\mathscr{A}(a)$ if and only if $f$ has an asymptotic value and every boundary path contains a sequence of points $a_{n}$ such that $\lim _{n \rightarrow \infty} f\left(z_{n}\right)=a$ and $\lim _{n \rightarrow \infty}\left|z_{n}\right|=1$

Proof. If $f \in \mathscr{A}(a)$ the conclusion follows immediately.

Let $f \notin \mathscr{A}(a)$. Then by Theorem 1 there exists a level curve $C(\lambda)$ of the function $f(z)-a$, or $f(z)$ if $a=\infty$, which is disjoint from the zeros of $f^{\prime}(z)$ and is not a Jordan curve in $D$. Hence, $C(\lambda)$ contains a boundary path $T$ on which $|f(z)-a|=\lambda$, or $|f(z)|=\lambda$ if $a=\infty$, and so there does not exist a sequence $z_{n} \in T$ such that $\lim _{n \rightarrow \infty}$ $f\left(z_{n}\right)=a$ and $f$ fails to satisfy the conditions of the corollary. This completes the proof. 
4. A short proof of a corollary of McMillan. The proof given in this section is elementary in the sense that it uses only the classical results of Fatou and F. and M. Riesz. The theorem of McMillan $[4, p .151]$ to which this corollary refers is very complicated and uses many measure theoretic concepts.

The method of proof uses a result of MacLane [3, p. 13] which was used to prove several results in [7].

Definition 7. The end of the tract $\{D(\varepsilon), a\}$ is $\bigcap_{\varepsilon>0} \bar{D}(\varepsilon)$ where $\bar{D}(\varepsilon)$ represents the closure of $D(\varepsilon)$.

THEOREM 2 (McMillan). If $f(z)$ is a holomorphic function in $D$ which has a finite number of tracts, the union $K$ of the ends of the tracts associated with $\infty$ is the circle $C:|z|=1$.

Proof. Let $T_{1}(\infty)=\left\{D_{1}(\varepsilon), \infty\right\}, T_{2}(\infty)=\left\{D_{2}(\varepsilon), \infty\right\}, \cdots, T_{m}(\infty)=$ $\left\{D_{m}(\varepsilon), \infty\right\}, T_{1}\left(a_{1}\right), T_{2}\left(a_{2}\right), \cdots, T_{p}\left(a_{p}\right)$, be the tracts for $f$ where $a_{i} \neq \infty, i=$ $1,2, \cdots, p$.

Assume the contrary of the conclusion: that is $K \neq C$. Because $K$ is closed there exists a disk $N$ about a point of $C$ such that $K \cap$ $\bar{N}=\phi$. If for some $i$ and every $\varepsilon>0, \bar{D}_{i}(\varepsilon) \cap \bar{N} \neq \phi$, where $D_{i}(\varepsilon)$ is the set of domains for $T_{i}(\infty)$, select a sequence $\left\{\varepsilon_{n}\right\}$ with $\varepsilon_{n} \downarrow 0$ and a sequence $\left\{z_{n}\right\}$ such that $z_{n} \in \bar{D}_{i}\left(\varepsilon_{n}\right) \cap \bar{N}$. By Definition $6\left\{z_{n}\right\}$ has a limit point $\zeta \in C$ which is also in $\bar{N}$. Then $\zeta \in \bigcap_{j=1}^{\infty} \bar{D}_{i}\left(\varepsilon_{j}\right) \subset K$, in violation of $K \cap \bar{N}=\phi$. Thus for each $n=1,2, \cdots, m$ there exists an $\varepsilon_{n}>0$ such that $\bar{D}_{n}\left(\varepsilon_{n}\right) \cap \bar{N}=\phi$. For $\varepsilon=\operatorname{Min}\left\{\varepsilon_{1}, \varepsilon_{2}, \cdots, \varepsilon_{m}\right\}$, we have $\bar{D}_{n}(\varepsilon) \cap \bar{N}=\phi$, for each $n, n=1,2, \cdots, m$.

If $f(z)$ were bounded in $N \cap D$, then $f(z)$ has radial limit almost everywhere on $N \cap C$ by the theorem of Fatou [2]. These limits must be selected from $\left\{a_{1}, a_{2}, \cdots, a_{p}\right\}$. Let $A_{i}$ be the set of $\zeta \in N \cap C$ for which $f$ has radial limit $a_{i}$. By the F. and M. Riesz theorem [5] the measure of $A_{i}$ is 0 . Hence the measure of $\bigcup_{i=1}^{p} A_{i}$ is also 0 and $f(z)$ has radial limit on at most a set of measure 0 on $N \cap C$. Thus $f(z)$ is unbounded in $N \cap D$ and it is possible to choose $z_{0} \in N \cap D$ such that $\left|f\left(z_{0}\right)\right|>1 / \varepsilon, f^{\prime}\left(z_{0}\right) \neq 0$, and $f\left(z_{0}\right) \neq a_{i}$ for $i=1,2, \cdots, p$.

By methods of MacLane [3, p. 13] there exists an arc $T$ from $f\left(z_{0}\right)$ on the Riemann surface of $f^{-1}$ which ends at $\infty$. The arc $T$ can be chosen so that its projection in the $w$-plane is a ray on which $|w| \geqq\left|f\left(z_{0}\right)\right|$. The inverse image of $T$ has a component $\gamma$ which contains $z_{0}$. Because $|f(z)| \geqq\left|f\left(z_{0}\right)\right| \geqq 1 / \varepsilon$ for all $z \in \gamma$ and because $\gamma$ is an asymptotic path with $\infty$ as asymptotic value, $\gamma \subset D_{n}(\varepsilon)$ for some $n$ between 1 and $m$. This implies $z_{0} \in D_{n}(\varepsilon) \cap N$. But it has been established that $D_{n}(\varepsilon) \cap N=\phi$. This contradiction implies that the assumption $K \neq C$ is false and the theorem is proved. 
REMARK. The proof just given goes through for holomorphic functions with finite tracts associated with $\infty$ and a countable number of tracts associated with finite values. By another corollary of McMillan [4, p. 151] no such function exists. If $f(z)$ has finite tracts associated with $\infty$ and infinite tracts, then $f$ has point asymptotic values (values which are approached along a path that ends at a point of $C$ ) on a set of positive measure.

\section{REFERENCES}

1. F. Bagemihl, and P. Erdös, A problem concerning the zeros of a certain kind of holomorphic function in the unit disk, J. reine. angew. Math., 214/215 (1964), 340-344. 2. P. Fatou, Séries trigonométrique et séries de Taylor, Acta Math., 30 (1906), 335400 .

3. G. MacLane, Asymptotic values of holomorphic functions, Rice Institute Studies, 49 (1963).

4. J. E. McMillan, Asymptotic values of holomorphic functions, Mich. Math. J., 12 (1965), 141-154.

5. F. Riesz, and M. Riesz, Über die Randwerte einer analytischen Function, 4. Congr. Math. Scand. Stockholm, (1916), 27-47.

6. J. Warren, Annular functions, J. reine. angew. Math., 245 (1970), 143-148.

7. - Non-constant meromorphic functions and sequences of arcs, J. reine. angew. Math., (to appear).

Received August 19, 1970.

Pennsylvania State University 


\section{PACIFIC JOURNAL OF MATHEMATICS}

\section{EDITORS}

\author{
H. SAMELSON \\ Stanford University \\ Stanford, California 94305 \\ C. R. HовBY \\ University of Washington \\ Seattle, Washington 98105
}

J. DUGUndis

Department of Mathematics

University of Southern California

Los Angeles, California 90007

RICHARD ARENS

University of California

Los Angeles, California 90024

\section{ASSOCIATE EDITORS}
E. F. BeCKenbaCH
B. H. NeUmanN
F. WOLF
K. YoshidA

\section{SUPPORTING INSTITUTIONS}

\author{
UNIVERSITY OF BRITISH COLUMBIA \\ CALIFORNIA INSTITUTE OF TECHNOLOGY \\ UNIVERSITY OF CALIFORNIA \\ MONTANA STATE UNIVERSITY \\ UNIVERSITY OF NEVADA \\ NEW MEXICO STATE UNIVERSITY \\ OREGON STATE UNIVERSITY \\ UNIVERSITY OF OREGON \\ OSAKA UNIVERSITY \\ UNIVERSITY OF SOUTHERN CALIFORNIA
}

\author{
STANFORD UNIVERSITY \\ UNIVERSITY OF TOKYO \\ UNIVERSITY OF UTAH \\ WASHINGTON STATE UNIVERSITY \\ UNIVERSITY OF WASHINGTON

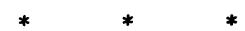 \\ AMERICAN MATHEMATICAL SOCIETY \\ CHEVRON RESEARCH CORPORATION \\ NAVAL WEAPONS CENTER
}

The Supporting Institutions listed above contribute to the cost of publication of this Journal, but they are not owners or publishers and have no responsibility for its content or policies.

Mathematical papers intended for publication in the Pacific Journal of Mathematics should be in typed form or offset-reproduced, (not dittoed), double spaced with large margins. Underline Greek letters in red, German in green, and script in blue. The first paragraph or two must be capable of being used separately as a synopsis of the entire paper. The editorial "we" must not be used in the synopsis, and items of the bibliography should not be cited there unless absolutely necessary, in which case they must be identified by author and Journal, rather than by item number. Manuscripts, in duplicate if possible, may be sent to any one of the four editors. Please classify according to the scheme of Math. Rev. Index to Vol. 39. All other communications to the editors should be addressed to the managing editor, Richard Arens, University of California, Los Angeles, California, 90024.

50 reprints are provided free for each article; additional copies may be obtained at cost in multiples of 50 .

The Pacific Journal of Mathematics is published monthly. Effective with Volume 16 the price per volume (3 numbers) is $\$ 8.00$; single issues, $\$ 3.00$. Special price for current issues to individual faculty members of supporting institutions and to individual members of the American Mathematical Society: $\$ 4.00$ per volume; single issues $\$ 1.50$. Back numbers are available.

Subscriptions, orders for back numbers, and changes of address should be sent to Pacific Journal of Mathematics, 103 Highland Boulevard, Berkeley, California, 94708.

PUBLISHED BY PACIFIC JOURNAL OF MATHEMATICS, A NON-PROFIT CORPORATION

Printed at Kokusai Bunken Insatsusha (International Academic Printing Co., Ltd.), 7-17, Fujimi 2-chome, Chiyoda-ku, Tokyo, Japan. 


\section{Pacific Journal of Mathematics}

Vol. 38, No. $2 \quad$ April, 1971

Richard Davis Anderson and Thomas Ashland Chapman, Extending

homeomorphisms to Hilbert cube manifolds .................. 281

Nguyen Huu Anh, Restriction of the principal series of $\operatorname{SL}(n, \mathbf{C})$ to some

reductive subgroups................................ 295

David W. Boyd, Indices for the Orlicz spaces . . . . . . . . . . . . 315

William Garfield Bridges, The polynomial of a non-regular digraph ...... 325

Billie Chandler Carlson, Robert K. Meany and Stuart Alan Nelson, Mixed

arithmetic and geometric means........................ 343

H. A. Çelik, Commutative associative rings and anti-flexible rings ...... 351

Hsin Chu, On the structure of almost periodic transformation groups ...... 359

David Allyn Drake, The translation groups of n-uniform translation

Hjelmslev planes ................................ 365

Michael Benton Freeman, The polynomial hull of a thin two-manifold . . . . 377

Anthony Alfred Gioia and Donald Goldsmith, Convolutions of arithmetic

functions over cohesive basic sequences .................... 391

Leslie C. Glaser, A proof of the most general polyhedral Schoenflies

conjecture possible ................................

Thomas Lee Hayden and Ted Joe Suffridge, Biholomorphic maps in Hilbert

space have a fixed point ................................ 419

Roger Alan Horn, Schlicht mappings and infinitely divisible kernels ...... 423

Norman Ray Howes, On completeness ...................... 431

Hideo Imai, Sario potentials on Riemannian spaces................ 441

A. A. Iskander, Subalgebra systems of powers of partial universal

algebras.

Barry E. Johnson, Norms of derivations of $\mathscr{L}(\mathrm{X})$.

David Clifford Kay and Eugene W. Womble, Axiomatic convexity theory and relationships between the Carathéodory, Helly, and Radon numbers

Constantine G. Lascarides, A study of certain sequence spaces of Maddox

and a generalization of a theorem of Iyer .............

C. N. Linden, On Blaschke products of restricted growth .

John S. Lowndes, Some triple integral equations ................. 515

Declan McCartan, Bicontinuous preordered topological spaces ......... 523

S. Moedomo and J. Jerry Uhl, Jr., Radon-Nikodým theorems for the Bochner and Pettis integrals ...

Calvin Cooper Moore and Joseph Albert Wolf, Totally real representations

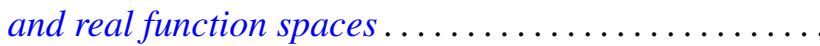

Reese Trego Prosser, A form of the moment problem for Lie groups. ... 